\title{
Well ordering groups with no monotone arithmetic progressions
}

\author{
Gyula Károlyi Péter Komjáth
}

June 17, 2016

\begin{abstract}
Károlyi-Kós and Ardal-Brown-Jungić proved that every vector space over $\mathbb{Q}$ has an ordering with no monotone three term arithmetic progression (3-AP). We show that every solvable group has a well ordering with no monotone 6-AP, and each hypoabelian group has an ordering omitting monotone 5-APs. Finally, we prove that every group has a well ordering with no infinite monotone AP.
\end{abstract}

In what follows, in a group, we call an arithmetic progression of length $n$, in short, an $n$-AP, a set of the form $\left\{x, x d, \ldots, x d^{n-1}\right\}$ consisting of distinct elements. (Perhaps the expression geometric progression would be more correct, we use the name arithmetic progression as we mostly consider commutative groups.) If $<$ is an ordering of a group, then an $n$-AP $\left\{x, x d, \ldots, x d^{n-1}\right\}$ is monotone, if $x<x d<\cdots<x d^{n-1}$ holds. Erdös and Graham asked ([4], $\mathrm{p} 22$.) if there is an ordering of the additive group of $\mathbb{R}$ with no monotone 3-AP. This was answered in the affirmative by Károlyi and Kós (unpublished, but see [5], p. 325) and independently, by Ardal, Brown, and Jungić ([1]). It is quite possible that there are other people who have asked or proved this.

A common feature of the proofs is the presence of some steps which guarantee the existence of a certain ordering assuming the existence of like orderings on all finite subsets (compactness principles, e.g., König's infinity lemma). These principles cannot be used to give well orderings, therefore we ask if the ordering in the above theorem can be a well ordering.

Alas, there is an easy argument showing that the answer is "no" (see Observation 3 below). We therefore modify the question to the following: 
given a group $G$, does there exist a number $r$, such that $G$ has a well ordering admitting no monotone $r$-AP.

We show that every solvable group has a well ordering with no monotone 6-AP (Theorem 9). If a group is hypoabelian, i.e., the derived subgroups reach 1 in a transfinite number of steps, then there is an ordering with no monotone 5-AP (Theorem 10). For arbitrary groups we show that every group has a well ordering with no infinite monotone arithmetic progression (Theorem 11).

We mention that this kind of studies about avoidance patterns in algebraic studies was initiated in [2].

Conventions. We use the notation of axiomatic set theory, especially in the proof of Theorems 10 and 11 .

In a group $G$, for $r \leq \omega$ an $r$-AP, an arithmetic progression of length $r$ is $\operatorname{AP}_{r}(x, d)=\left\{x d^{i}: i<r\right\}$ assuming that the elements are different, that is, the order of $d$ is at least $r$. The apparent asymmetry in the definition is resolved by the fact that $x d^{i}=D^{i} x$ where $D=x d x^{-1}$. Note that if $A \subseteq G$ has an ordering (well ordering) with no monotone $r$-AP, then the same is true for any subset of $A$.

A group $G$ is hypoabelian if the transfinitely extended derived series reaches 1, i.e., if $G_{0}=G, G_{\alpha+1}=G_{\alpha}^{\prime}, G_{\alpha}=\bigcap\left\{G_{\beta}: \beta<\alpha\right\}$ for $\alpha$ limit, then $G_{\alpha}=\{1\}$ for some ordinal $\alpha$. We refer to the smallest such $\alpha$ as the length of $G$. Obviously each solvable group (in fact each residually solvable group) is hypoabelian and so is every free group by a theorem of Magnus ([6]).

When we consider ordered or well ordered groups we do not assume any connection between the order and the operation, so these are not ordered structures in the usual sense.

In what follows, the well ordered groups will always have 1 (or 0 , if the operation is written additively) as the smallest element. This is nothing extra: if $<$ is some well ordering of a group $G$ with no monotone $r$-AP with $b$ as the smallest element, we define $x<y$ if and only if $b x<b y$. Now $<^{\prime}$ is a well ordering of $G$ (as they are order-isomorphic) and $\operatorname{AP}_{r}(a, d)$ is a monotone $r$-AP under $<^{\prime}$ if and only if $\mathrm{AP}_{r}(b a, d)$ is so under $<$.

For the notions of ultrafilter and ultraproduct, we refer to [3] or any textbook on model theory.

Lemma 1. If $n$ is a natural number, then $A_{n}=\{0,1, \ldots, n-1\}$ has an ordering $<_{n}$ with no monotone $3-A P$.

Proof. By induction on $n$. The case $n=1$ is obvious. 
It is enough to show that if the statement holds for $n$, then it holds for $2 n$. Decompose $A_{2 n}$ as $B \cup C$ where $B=\{0,2, \ldots, 2 n-2\}, C=\{1,3, \ldots, 2 n-1\}$. Define $<_{2 n}$ on $B$ and $C$ by $2 i<_{2 n} 2 j$ if and only if $2 i+1<_{2 n} 2 j+1$ if and only if $i<_{n} j$. Finally, place $C$ after $B$ by defining $b<_{2 n} c$ for each pair $b \in B, c \in C$.

In order to show that $<_{2 n}$ is as required, assume that $K \subset A_{2 n}$ is a monotone 3-AP under $<_{2 n}$. For parity reasons we cannot have that $|K \cap B|=$ 1 and $|K \cap C|=2$ or $|K \cap B|=2$ and $|K \cap C|=1$. On the other hand, were $K \subseteq B$ or $K \subseteq C$, we would get a 3 -AP in $A_{n}$, which is monotone under $<_{n}$, a contradiction.

Corollary 2. Each finite subset of $\mathbb{Q}$ has an ordering with no monotone 3-AP.

Proof. Note that a subset $S$ of $\mathbb{Q}$ has an ordering with no monotone 3-AP if and only if $a+b S:=\{a+b s \mid s \in S\}$, where $a, b \in \mathbb{Q}$ and $b \neq 0$, has such an ordering. Since each finite subset of $\mathbb{Q}$ is contained in $a+b A_{n}$ for some $n \in \mathbb{N}$ and $a, b \in \mathbb{Q}, b \neq 0$, the statement follows from Lemma 1.

Observation 3. If a group $G$ has no element of degree 2, then every well ordering of $G$ contains a monotone 3-AP.

Proof. Let $\prec$ be a well ordering of $G$. Let $x$ be the least, $y$ the second least element of $G$. Write $y$ in the form $y=x d$. Then $x d^{2} \notin\{x, x d\}$ so we must have $x \prec x d \prec x d^{2}$, and we found a monotone 3-AP.

Notice that specifically, if $n>1$ is odd, then Observation 3 implies that every ordering of $\left(\mathbb{Z}_{n},+\right)$ contains a monotone 3 -AP. The same is true in general if $n$ is not a power of 2, for any such group contains a nontrivial cyclic subgroup of odd order.

Lemma $4 . \mathbb{Z}_{n}$ has an ordering with no monotone $r$-AP, where

(a) $r=3$, if $n$ is a power of 2 ,

(b) $r=5$, otherwise.

Proof. (a) Write $\mathbb{Z}_{n}=\{0,1, \ldots, n-1\}$ where " $i$ " actually stands for the residue class modulo $n$ that contains the integer $i$. The proof of Lemma 1 goes through verbatim.

(b) Set $A=\left\{0, \ldots,\left\lfloor\frac{n-1}{2}\right\rfloor\right\}, B=\left\{\left\lceil\frac{n}{2}\right\rceil, \ldots, n-1\right\}$. Let $<_{n}$ be as in Lemma 1 , and define $<$ to be the ordering of $A_{n}$ such that $<\left.\right|_{A}=<\left._{n}\right|_{A},<\left.\right|_{B}=<\left._{n}\right|_{B}$ and $A<B$, that is, $a<b$ if $a \in A, b \in B$. 
We show that the order $<$, if considered an order of the residue classes $\bmod n$, has no monotone 5 -AP. Let $K$ be a putative monotone 5 -AP. We either have $|K \cap A| \geq 3$ or $|K \cap B| \geq 3$. As $A<B$, this implies that either $A$ or $B$ contains a monotone 3 -AP, $\{a, b, c\}$ with $a<b<c$. There is a fine point here, namely that $a, b, c$ only form a 3 -AP $\bmod n$, that is, $a+c \equiv 2 b \quad(\bmod n)$. As either $a, b, c \in A$ or $a, b, c \in B$, we have either $a+c, 2 b \in\{1, \ldots, n-1\}$ or $a+c, 2 b \in\{n+1, \ldots, 2 n-2\}$, and this, together with $a+c \equiv 2 b \quad(\bmod n)$, implies $a+c=2 b$, so $a, b, c$ form a monotone 3 -AP also under $<_{n}$ in $A_{n}$, contradicting Lemma 1.

Lemma 5. Let $G=\mathbb{Q}$ or $\mathbb{Z}_{2^{\infty}}$ and $r=3$, or else let $G=\mathbb{Z}_{p^{\infty}}(p$ an odd prime) and $r=5$. Then there is an ordering of $G$ with no monotone $r-A P$.

Proof. Each finite subset of $\mathbb{Z}_{p \infty}$ is contained in a finite subgroup isomorphic to $\mathbb{Z}_{p^{n}}$ for some $n$. Therefore we can apply either Corollary 2 (when $G=\mathbb{Q}$ ) or else Lemma 4 to conclude that each finite subset of $G$ has an ordering with no monotone $r$-AP. One can either refer to König's infinity lemma or Rado's selection lemma ([7]) to extend this property to $G$.

Lemma 6. If $N \triangleleft G$ and both $N$ and $G / N$ have orderings (well orderings) omitting monotone $r$-APs, then so has $G$.

Proof. Let $\varphi: G \rightarrow G / N$ be the factor homomorphism. Decompose $G$ into cosets of $N$ :

$$
G=\bigcup_{i \in G / N} r_{i} N
$$

with $r_{1}=1$. Let $<_{N}$, resp. $<_{G / N}$ be orderings of $N$ and $G / N$ omitting monotone $r$-APs. We define the following relation $\prec$ on $\mathrm{G}$. If $x=r_{i} x^{\prime}$ where $i=\varphi(x)$ and $x^{\prime} \in N, y=r_{j} y^{\prime}$ where $j=\varphi(y)$ and $y^{\prime} \in N$, then set $x \prec y$ if and only if either $i=j$ and $x^{\prime}<_{N} y^{\prime}$ or $i<_{G / N} j$.

It is immediately seen that $\prec$ is an ordering and it is a well ordering if both $<_{N}$ and $<_{G / N}$ are.

Assume that $x \prec x d \prec x d^{2} \prec \cdots \prec x d^{r-1}$ is a monotone $r$-AP. There are two cases to distinguish.

Case 1. $d \in N$.

If now $x=r_{i} x^{\prime}$ where $i=\varphi(x)$, then $x, x d, \ldots, x d^{r-1} \in r_{i} N$ and by the way $\prec$ was defined we must have $x^{\prime}<_{N} x^{\prime} d<_{N} \cdots<_{N} x^{\prime} d^{r-1}$, contradiction.

Case 2. $d \notin N$. 
In this case $x d^{j}$ and $x d^{j+1}$ lie in different cosets of $N$ for every $j \in$ $\{0,1, \ldots, r-2\}$. Thus, $\varphi\left(x d^{j}\right)<_{G / N} \varphi\left(x d^{j+1}\right)$ and we must have

$$
\varphi(x)<_{G / N} \varphi(x) \varphi(d)<_{G / N} \cdots<_{G / N} \varphi(x) \varphi(d)^{r-1},
$$

a contradiction to the assumption that there is no monotone $r$-AP in $G / N$ under $<_{G / N}$.

Remark 7. It is seen from the above proof that if $<_{N}$ and $<_{G / N}$ are well orderings of $N$, resp. $G / N$ with the unit element as the smallest, then $<_{G}$ has a similar property. Moreover, $<_{G}$ end extends $<_{N}$, that is, $<_{N}=<\left._{G}\right|_{N}$ and $n<_{G} g$ holds for every pair $n \in N, g \in G \backslash N$.

Lemma 8. Let $G=\mathbb{Q}$ and $r=6$, or $G=\mathbb{Z}_{2^{\infty}}$ and $r=3$, or $G=\mathbb{Z}_{p^{\infty}}$ ( $p$ an odd prime) and $r=5$. Then the group $G$ has a well ordering with no monotone $r-A P$.

Proof. If $G=\mathbb{Z}_{p^{\infty}}$, then $G$ is the increasing union of the subgroups $G_{n}$ such that $G_{n} \simeq \mathbb{Z}_{p^{n}}, G_{n+1} / G_{n} \simeq \mathbb{Z}_{p}$. Using Lemmas 4 and 6 and Remark 7 , we can define the ordering $<_{n}$ of $G_{n}$ with no monotone $r$-AP, such that $<_{n+1}$ end extends $<_{n}$, and so the union of the orderings is a well ordering of $\mathbb{Z}_{p^{\infty}}$ with no monotone $r$-AP. Note that in the case $p=2$ this is exactly what happened in the proof of Lemma 4(a).

For $G=\mathbb{Q}$ we proceed as follows. Enumerate $\mathbb{Q}$ as $\mathbb{Q}=\left\{q_{0}, q_{1}, \ldots\right\}$ with $q_{0}=0$. Define the finite subsets $V_{0} \subseteq V_{1} \subseteq \cdots$ such that

(1) $V_{0}=\left\{q_{0}\right\}$,

(2) $q_{n} \in V_{n}$,

(3) if $K$ is a 6 -AP with $\left|K \cap V_{n}\right| \geq 2$, then $K \subseteq V_{n+1}$,

(4) $V_{n+1}$ is minimal with respect to (2) and (3).

Notice that $V_{n+1}$ is finite as $V_{n}$ is finite and there are exactly 15 ways to extend a 2-element subset of $\mathbb{Q}$ to a 6 -set whose elements form a 6 -AP, so only finitely many 6 -APs are to be considered in (3). By (2), we have $\bigcup\left\{V_{n}: n<\omega\right\}=\mathbb{Q}$. By Corollary 2, there is an ordering $\prec_{n}$ of $V_{n} \backslash V_{n-1}$ with no monotone 3-AP. Define the ordering $\prec$ of $\mathbb{Q}$ as follows. If $x \neq y$, let $x \prec y$ if either $x, y \in V_{n} \backslash V_{n-1}$ and $x \prec_{n} y$ for some $n$, or else $x \in V_{n}$, $y \in V_{n+1} \backslash V_{n}$ for some $n$. That is, $\prec$ agrees with $\prec_{n}$ on each $V_{n} \backslash V_{n-1}$ and 
we place the sets $V_{0}, V_{1} \backslash V_{0}, V_{2} \backslash V_{1}, \ldots$ one after the other. Obviously, $\prec$ is a well ordering of $\mathbb{Q}$ into type $\omega$ with 0 as the least element.

Assume now that $K$ is a monotone 6 -AP. Let $n$ be minimal so that $\mid K \cap$ $V_{n+1} \mid \geq 2$ holds. Then $\left|K \cap V_{n}\right| \leq 1$ by the minimality of $n$, and $K \subseteq V_{n+2}$ by the above construction. Notice that $K \cap\left(V_{n+1} \backslash V_{n}\right)$ and $K \cap\left(V_{n+2} \backslash V_{n+1}\right)$ are segments of the arithmetic progression $K$, and so they are arithmetic progressions themselves. Further, $\left|K \cap\left(V_{n+1} \backslash V_{n}\right)\right| \leq 2$ and $\mid K \cap\left(V_{n+2} \backslash\right.$ $\left.V_{n+1}\right) \mid \leq 2$, as otherwise there would be a monotone 3-AP in either $V_{n+1} \backslash V_{n}$ or $V_{n+2} \backslash V_{n+1}$. Adding up, we obtain

$$
|K|=\left|K \cap V_{n}\right|+\left|K \cap\left(V_{n+1} \backslash V_{n}\right)\right|+\left|K \cap\left(V_{n+2} \backslash V_{n+1}\right)\right| \leq 5<|K| .
$$

This contradiction completes the proof.

Theorem 9. Every solvable group has a well ordering with no monotone $6-A P$.

Proof. Lemma 6 reduces the statement to the case of abelian groups. As every abelian group can be embedded into a divisible group (see [8]), it suffices to prove the results for divisible groups.

Let $G$ be a divisible group. By a well known result $G$ can be written as the direct sum of some groups $G_{i}(i \in I)$, where each $G_{i}$ is isomorphic to either $\mathbb{Q}$ or $\mathbb{Z}_{p^{\infty}}$ for some prime $p$, cf. [8]. By Lemma 8, there is a well ordering $<_{i}$ of $G_{i}$ with no monotone 6 -AP, such that 0 is the least element of $G_{i}$ under $<_{i}$. Let $<$ be a well ordering of $I$. Then, each element $x$ of $G$ can be written as a sequence $x=(x(i): i \in I)$ where $x(i) \in G_{i}$ and $\operatorname{supp}(x)=\{i \in I: x(i) \neq 0\}$ is finite. Order $G$ by last comparison, that is, if $x=(x(i): i \in I)$ and $y=(y(i): i \in I)$, then set

$$
d(x, y)=\max _{<}\{i \in I: x(i) \neq y(i)\}
$$

and let $x \prec y$ if and only if $x(i)<_{i} y(i)$ holds for $i=d(x, y)$.

Claim 1. $\prec$ is an ordering.

Proof. Assume that $x \prec y$ and $y \prec z$. Set $i=d(x, y), j=d(y, z)$.

If $i=j$, then $x(i)<_{i} y(i)<_{i} z(i)$, therefore $x(i)<_{i} z(i)$ and $d(x, z)=i$ and so $x \prec z$.

If $i<j$, then $x(j)=y(j)<_{i} z(j)$ and clearly $d(x, z)=j$, therefore $x \prec z$.

If $j<i$, then $x(i)<_{i} y(i)=z(i)$, so $d(x, z)=i$ and therefore $x \prec z$.

Claim 2. $\prec i s$ a well ordering. 
Proof. Assume that $x_{0}, x_{1}, \ldots$ is a $\prec$-decreasing sequence of elements of $G$. We color the triple $\left\{j_{0}, j_{1}, j_{2}\right\}$ of natural numbers with $j_{0}<j_{1}<j_{2}$ by three colours according to whether $d\left(x_{i_{0}}, x_{j_{1}}\right)>d\left(x_{j_{1}}, x_{j_{2}}\right), d\left(x_{j_{0}}, x_{j_{1}}\right)=$ $d\left(x_{j_{1}}, x_{j_{2}}\right)$, or $d\left(x_{j_{0}}, x_{j_{1}}\right)<d\left(x_{j_{1}}, x_{j_{2}}\right)$ holds. Applying Ramsey's theorem we obtain an infinite homogeneous set, therefore a subsequence $y_{0}, y_{1}, \ldots$ such that either $d\left(y_{j}, y_{j+1}\right)>d\left(y_{j+1}, y_{j+2}\right)$ or $d\left(y_{j}, y_{j+1}\right)=d\left(y_{j+1}, y_{j+2}\right)$ or $d\left(y_{j}, y_{j+1}\right)<d\left(y_{j+1}, y_{j+2}\right)$ holds for every nonnegative integer $j$.

The first possibility is impossible as it would give an infinite decreasing sequence in the well ordered set $I$.

If the second possibility holds, i.e., if $d\left(y_{0}, y_{1}\right)=d\left(y_{1}, y_{2}\right)=\cdots=i$, then $y_{0}(i), y_{1}(i), \ldots$ would be an infinite $<_{i}$-decreasing sequence, a contradiction, as $<_{i}$ is a well order of $G_{i}$.

Assume now the third possibility, i.e., that $i_{0}<i_{1}<\cdots$ where $i_{j}=$ $d\left(y_{j}, y_{j+1}\right)$. For each $j$, as $y_{j}\left(i_{j}\right)>_{i_{j}} y_{j+1}\left(i_{j}\right)$ holds, we infer $y_{j}\left(i_{j}\right) \neq 0$ (as 0 is the least element of $\left.G_{i_{j}}\right)$, and as $d\left(y_{0}, y_{1}\right), d\left(y_{1}, y_{2}\right), \ldots, d\left(y_{j-1}, y_{j}\right)<i_{j}$, we have

$$
y_{0}\left(i_{j}\right)=y_{1}\left(i_{j}\right)=\cdots=y_{j}\left(i_{j}\right) \neq 0,
$$

and so $\left\{i_{0}, i_{1}, \ldots\right\} \subseteq \operatorname{supp}\left(y_{0}\right)$, contradicting the finiteness of $\operatorname{supp}\left(y_{0}\right)$.

Claim 3. There is no monotone 6 -AP under $\prec$.

Proof. Assume that $y_{0} \prec y_{1} \prec \cdots \prec y_{5}$ is a monotone 6-AP. Let $j$ denote the largest element of $I$ for which $y_{0}(j), \cdots, y_{5}(j)$ is not constant; it exists by the finiteness of $\operatorname{supp}\left(y_{0}\right), \ldots, \operatorname{supp}\left(y_{5}\right)$. For each $i \in I$, either $y_{0}(i)=\cdots=y_{5}(i)$, or $y_{0}(i), \cdots, y_{5}(i)$ form a proper 6 -AP in $G_{i}$, or it a non-constant 6 -AP in which some repetitions occur. However, by the monotonicity of $y_{0}, y_{1}, \ldots, y_{5}$ under $\prec$, only the second possibility can happen for $i=j$. Thus, $d\left(y_{0}, y_{1}\right)=$ $\cdots=d\left(y_{4}, y_{5}\right)=j$ and $y_{0}(j)<_{j} \cdots<_{j} y_{5}(j)$ is a monotone 6-AP in $G_{j}$, a contradiction.

With Claims 1-3, the proof of the statement on well ordering of the group is complete.

Theorem 10. Each hypoabelian group has an ordering with no monotone 5-AP.

Proof. We prove the statement by transfinite induction on the length of $G$, denoted by $\alpha$. If $\alpha \leq 1$, that is, the group $G$ is abelian, then we may proceed as in the proof of Theorem 9, using Lemma 5 instead of Lemma 8, and merely referring to Claims 1 and 3. In fact, in this case, lexicographic ordering of the direct sum along the well ordered index set $I$ would also do. 
Let now $\alpha>1$, and assume that we have already proved the statement for all ordinals less than $\alpha$. We distinguish two cases.

Case 1. $\alpha=\beta+1$ is a successor ordinal.

By the inductive hypothesis, there is an ordering, as required, on $G / G_{\beta}$, and also on the abelian group $G_{\beta}$. We conclude by Lemma 6 .

Case 2. $\alpha$ is a limit ordinal.

In this case $G_{\alpha}=\bigcap\left\{G_{\beta}: \beta<\alpha\right\}=\{1\}$ and each $G / G_{\beta}$ has an ordering $<_{\beta}$ omitting monotone 5-APs. Note that, in the first order language of groups equipped with a linear order, the fact that a given structure does not contain a monotone 5-AP can be expressed by a first order sentence $\Phi$.

Let $U$ be an ultrafilter on $\alpha$ such that the terminal interval $[\beta, \alpha)$ is in $U$ for each $\beta<\alpha$, and consider the ultraproduct

$$
\left(G^{*},<^{*}\right)=\prod_{\beta<\alpha}\left(G / G_{\beta},<_{\beta}\right) / U .
$$

By Loś's lemma, $\left(G^{*},<^{*}\right) \models \Phi$. It is enough to show that $G$ embeds into $G^{*}$.

For this, define $j(x)$ as the equivalence class $[f]$ where $f(\beta)$ is the coset of $G_{\beta}$ containing $x . j: G \rightarrow G^{*}$ is obviously a homomorphism, and if $x \neq 1$, then $x G_{\beta} \neq G_{\beta}$ for $\beta$ sufficiently large, so by the choice of $U$ we have $j(x) \neq 1$. Consequently, $j$ is an embedding.

Theorem 11. Every group has a well ordering with no monotone infinite arithmetic progressions.

Proof. First we prove that if $G$ is a group, then there is a system

$$
\mathcal{H}(G)=\left\{\left\langle A_{i},<_{i}\right\rangle: i \in I\right\}
$$

of ordered subsets such that $\left|A_{i}\right|=\omega, A_{i} \cap A_{j}=\emptyset(i \neq j)$, and if $A=$ $\operatorname{AP}_{\omega}(x, d)=\left\{x, x d, x d^{2}, \ldots\right\}$ is an infinite AP in $G$, then there is an $i$ such that $A_{i} \subseteq A$ and $x d^{a}<_{i} x d^{b}$ holds, whenever $a<b$ and $x d^{a}, x d^{b} \in A_{i}$.

We prove this statement by induction on the cardinal $\kappa=|G|$. Let $\mathcal{H}(G)=\emptyset$ if $G$ is finite.

If $\kappa=\omega$, there are countably many infinite arithmetic progressions. These are countably many infinite sets, we can find pairwise disjoint infinite subsets $A_{i}$ of them by an inductive argument (cf. Problem 12.2. in [5], p. 59) and make $<_{i}$ the inherited ordering.

Assume now that $\kappa>\omega$. Decompose $G$ as the increasing union $G=$ $\bigcup\left\{G_{\alpha}: \alpha<\kappa\right\}$ of subgroups such that $\left|G_{\alpha}\right|<\kappa(\alpha<\kappa), G_{0}=\{1\}$, and 
$G_{\alpha}=\bigcup\left\{G_{\beta}: \beta<\alpha\right\}$ for $\alpha<\kappa$ limit, i.e., $\left\{G_{\alpha}: \alpha<\kappa\right\}$ is continuous. This can be done for example by enumerating the elements of $G$ as $\left\{g_{\alpha}: \alpha<\kappa\right\}$ and letting $G_{\alpha}$ be the subgroup generated by $\left\{g_{\beta}: \beta<\alpha\right\}$.

Set $\mathcal{H}_{\alpha}=\mathcal{H}\left(G_{\alpha+1}\right)$, i.e., a system as described above, for the group $G_{\alpha+1}$. It exists by the inductive assumption.

For each $A \in \mathcal{H}_{\alpha}$, set $A^{*}=A \backslash G_{\alpha}$ together with the ordering inherited from $A$ if $\left|A \cap G_{\alpha}\right| \leq 1$, and undefined otherwise. Let $\mathcal{H}_{\alpha}^{*}=\left\{A^{*}: A \in \mathcal{H}_{\alpha}\right\}$. We claim that $\mathcal{H}(G)=\bigcup\left\{\mathcal{H}_{\alpha}^{*}: \alpha<\kappa\right\}$ satisfies the requirements.

First, each element of $\mathcal{H}(G)$ is of the form $A^{*}$ for some $A \in \mathcal{H}_{\alpha}, \alpha<\kappa$, therefore $\left|A^{*}\right|=|A|=\omega$.

Next, any two elements of $\mathcal{H}(G)$ are disjoint. Indeed, suppose that $A^{*} \neq$ $B^{*}$ for some $A \in \mathcal{H}_{\alpha}, B \in \mathcal{H}_{\beta}, \alpha \leq \beta<\kappa$. If $\alpha=\beta$, then $A^{*} \cap B^{*}=\emptyset$ follows from the disjointness of the elements of $\mathcal{H}_{\alpha}$, and if $\alpha<\beta$, then $B^{*}$ is disjoint from $G_{\beta} \supseteq G_{\alpha+1} \supseteq A \supseteq A^{*}$.

Finally, let $A=\operatorname{AP}_{\omega}(x, d)$ be an infinite arithmetic progression in $G$. Let $\beta<\kappa$ be the smallest ordinal such that $\left|A \cap G_{\beta}\right| \geq 2$. As $\left\{G_{\alpha}: \alpha<\kappa\right\}$ is continuous, $\beta=\alpha+1$ for some $\alpha$.

If $x d^{a}, x d^{b} \in G_{\alpha+1}$, then $\left(x d^{a}\right)^{-1}\left(x d^{b}\right)=d^{b-a} \in G_{\alpha+1}$. Let $k$ be the smallest positive integer such that $d^{k} \in G_{\alpha+1}$. There is a unique $0 \leq r<k$ that $x d^{r} \in G_{\alpha+1}$. Then $\left\{a: x d^{a} \in G_{\alpha+1}\right\}=k \mathbb{Z}+r$. Then $B=\operatorname{AP}_{\omega}\left(x d^{r}, d^{k}\right) \subseteq$ $A \cap G_{\alpha+1}$, and the arithmetic progression $B$ is infinite as $d^{k}$ has no finite order. By the minimal choice of $\beta$ we have $\left|B \cap G_{\alpha}\right| \leq 1$, and so some $A_{i} \in \mathcal{H}_{\alpha}$ has $A_{i}^{*} \subseteq B \subseteq A$. The monotonicity being inherited from $A_{i}$, the proof of the claim is thus complete.

Given $\mathcal{H}=\left\{\left\langle A_{i},<_{i}\right\rangle: i \in I\right\}$ as above, we let $\prec$ be an arbitrary well ordering of $G$ such that $\left.\prec\right|_{A_{i}} \neq<_{i}(i \in I)$. Then $\prec$ is not monotone on any infinite arithmetic progression, and so we are done.

Open Problems. 1) Is it true, that every $\mathbb{Z}_{p}, p$ a prime, has an ordering with no monotone 4-AP? 2) Determine the smallest $r$ for which $\mathbb{Q}$ has a well ordering with no monotone $r$-AP. 3) Is there, for every group $G$, a finite number $r=r(G)$ such that $G$ has a well ordering with no monotone $r$-AP?

Acknowledgments. Part of this research was done when the first author visited the School of Mathematics and Physics at the University of Queensland, supported by the Australian Research Council and by the Hungarian National Research Grant OTKA K 100291.

We are grateful to the referee for a very careful job. 


\title{
References
}

[1] Ardal, H., Brown, T., Jungić, V.: Chaotic orderings of the rationals and the reals. Amer. Math. Monthly 118, 921-925 (2011)

[2] Davis, J.A., Entringer, R.C., Graham, R.L., Simmons, G.J.: On permutations containing no long arithmetic progressions, Acta Arith. 34, 81-90 $(1977 / 78)$

[3] Eklof, P.C.: Ultraproducts for algebraists. In: Barwise, J. (ed.) Handbook of Mathematical Logic. Studies in Logic and the Foundations of Mathematics 90, pp. 105-137. North-Holland (1977)

[4] Erdős, P., Graham, R.L.: Old and New Problems and Results in Combinatorial Number Theory. Monographies de l'Enseignement Mathématique 28. Université de Genève (1980)

[5] Komjáth, P., Totik, V.: Problems and Theorems in Classical Set Theory. Problem Books in Mathematics. Springer, New York (2006)

[6] Magnus, W.: Beziehungen zwischen Gruppen und Idealen in einem speziellen Ring, Math. Ann. 111, 259-280 (1935)

[7] Rado, R.: Axiomatic treatment of rank in infinite sets. Canadian J. Math. 1, 337-343 (1949)

[8] Robinson, D.J.S.: A Course in the Theory of Groups. Second edition. Graduate Texts in Mathematics 80. Springer, New York (1996)

\author{
Gyula Károlyi \\ Alfréd Rényi Institute of Mathematics \\ Hungarian Academy of Sciences \\ Budapest, P.O.Box 127 \\ 1364, Hungary \\ and \\ Institute of Mathematics \\ Eötvös University \\ Budapest, P.O.Box 120 \\ 1518, Hungary \\ e-mail: karolyi.gyula@renyi.mta.hu
}


Péter Komjáth

Institute of Mathematics

Eötvös University

Budapest, P.O.Box 120

1518, Hungary

e-mail: kope@cs.elte.hu 